\title{
PET Scanner 3D Mode
}

National Cancer Institute

\section{Source}

National Cancer Institute. PET Scanner 3D Mode. NCI Thesaurus. Code C94950.

A measuring process without any shielding septa restricting detectors' coincidences. It is generally referred to as simply 3D. 